\title{
Generalized Gaussian Jacobsthal and Generalized Gaussian Jacobsthal Lucas Polynomial
}

\section{Arun Kumar Yadav}

\begin{abstract}
The aim of the present paper is to present a generalization of Gaussian Jacobsthal polynomial and Gaussian Jacobsthal Lucas polynomial. Present paper extends the work of Asci and Gurel [3]. Some important generalizations of Generating function, Binet formula, Explicit formula, $Q$ matrix and determinantal representations of these polynomials are also produced.

Keywords: Generalized Gaussian Jacobsthal; Generalized Gaussian Jacobsthal Lucas Polynomial; Binet formula; Cassini Identity
\end{abstract}

\section{INTRODUCTION}

In 1977, Berzsenyi [4] initiated the theory of Gaussian Fibonacci numbers. Afterwards different authors formulated various interesting results using Gaussian Fibonacci and Gaussian Lucas polynomials. On the other hand, Asci and Gurel [1] introduced Bivariate Gaussian Fibonacci and Bivariate Gaussian Lucas polynomials. In the same work, authors [Asci and Gurel] gave generating functions, Binet formulas, explicit formulas and $Q$ matrix for these polynomials. Simillarly, Asci and Gurel [2] defined Gaussian Jacobsthal and Gaussian Jacobsthal Lucas numbers and using these notions they discussed some interesting results. Recently, Asci and Gurel [3] defined the Gaussian Jacobsthal polynomial and Gaussian Jacobsthal Lucas polynomials and formulated generating function, Binet formula, Explicit formulas and determinantal representations for these polynomials.

Horadam [7] introduced the Jacobsthal and the Jacobsthal Lucas sequences under the following recurrence relations:

$$
\mathrm{J}_{\mathrm{n}}=\mathrm{J}_{\mathrm{n}-1}+2 \mathrm{~J}_{\mathrm{n}-2}, \mathrm{n} \geq 2
$$

where $l_{0}=0$ and $l_{1}=1$, and

$$
j_{n}=j_{n-1}+2 j_{n-2}, n \geq 2
$$

where $\tilde{j}_{0}=2$ and $\tilde{j}_{1}=1$.

In the same work Horadam [7], Jacobsthal and the Jacobsthal Lucas polynomial sequences were defined by using the following recurrence relations:

$$
J_{n}(x)=J_{n-1}(x)+2 J_{n-2}(x), n \geq 2
$$

where $l_{0}=0$ and $l_{1}=1$, and

$$
j_{n}(x)=j_{n-1}(x)+2 j_{n-2}(x), n \geq 2
$$

where $j_{0}=2 \mathrm{x}$ and $j_{1}=1$.

Subsequently, the Gaussian Jacobsthal sequence and Gaussian Jacobsthal Lucas sequence were defined by Asci and Gurel [2] as follows:

Revised Manuscript Received on September 22, 2019.

* Correspondence Author

Arun Kumar Yadav*, Department of Mathematics, Ahir college, Rewari 123401, India
$\mathrm{GJ}_{\mathrm{n}+1}=G \mathrm{~J}_{\mathrm{n}}+2 \mathrm{GJ}_{\mathrm{n}-1}, \mathrm{n} \geq 1$

where $G J_{0}=\frac{i}{2}$ and $G J_{1}=1$, and

$\mathrm{G}_{\mathrm{n}+1}=G_{\mathrm{j}_{\mathrm{n}}}+2 \mathrm{G}_{\mathrm{n}-1}, \mathrm{n} \geq 1$

where $G_{j_{0}}=2-\frac{i}{2}$ and $G_{j_{1}}=1+2 i$.

The Gaussian Jacobsthal and Gaussian Jacobsthal Lucas polynomial in [3] were defined by

$$
\mathrm{GJ}_{\mathrm{n}+1}(\mathrm{x})=G \mathrm{~J}_{\mathrm{n}}(\mathrm{x})+2 \mathrm{GJ}_{\mathrm{n}-1}(\mathrm{x}), \mathrm{n} \geq 1
$$

where $G J_{0}(x)=\frac{i}{2}$ and $G J_{1}(x)=1$, and

$$
\mathrm{G}_{\mathrm{n}+1}^{\mathrm{i}}(\mathrm{x})=\mathrm{G}_{\mathrm{n}}(\mathrm{x})+2 \mathrm{G} \mathrm{j}_{\mathrm{n}-1}(\mathrm{x}), \mathrm{n} \geq 1
$$

where $\mathrm{G}_{0}(\mathrm{x})=2-\frac{\mathrm{i}}{\mathbf{2}}$ and $\mathrm{G}_{j_{1}}(\mathrm{x})=1+2 i$.

Further, in [3] the authors have also formulated the following notions:

Generating functions:

(i) $\mathrm{g}(\mathrm{t}, \mathrm{x})=\sum_{n=0}^{\mathrm{s}=0} \mathrm{G} \mathrm{I}_{\mathrm{n}}(\mathrm{x}) \cdot \mathrm{t}^{\mathrm{n}}=\frac{2 \mathrm{t}+\mathrm{i}(1-\mathrm{t})}{2-2 \mathrm{t}-4 \mathrm{t}^{2} x}$;

(ii) $\mathrm{h}(\mathrm{t}, \mathrm{x})=\sum_{n=0}^{\mathrm{m}} \mathrm{G} \mathrm{I}_{\mathrm{n}}(\mathrm{x}) \cdot \mathrm{t}^{n}=\frac{4-2 t+i(\mathrm{t}-1+4 x t)}{2-2 \mathrm{t}-4 \mathrm{t}^{2} x}$.

Binet Formula:

For $\mathrm{n} \geq 0$,

(i) $G J_{n}(x)=\frac{a^{n}(x)-\beta^{n}(x)}{a(x)-\beta(x)}+i \cdot x \frac{a^{n-1}(x)-\beta^{n-1}(x)}{\alpha(x)-\beta(x)}$;

(ii) $G_{j_{n}}(x)=\alpha^{n}(x)+\beta^{n}(x) \quad+$ i. $x \cdot\left(\alpha^{n-1}(x)+\beta^{n-1}(x)\right)$

Explicit Formula:

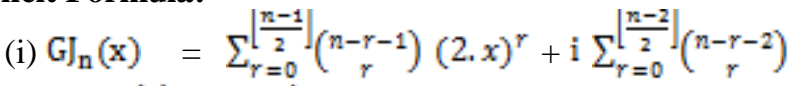

$$
\begin{aligned}
& (2)^{\gamma} \cdot(x)^{\gamma+1} \\
& \text { (ii) }
\end{aligned}
$$

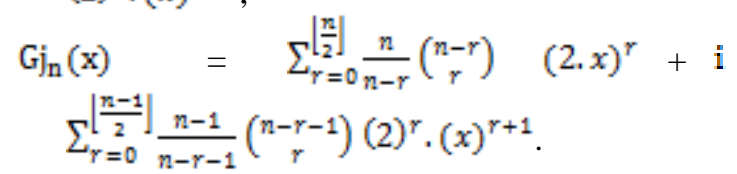

Cassini Identity:

For $\mathrm{n} \geq 1$,

$$
\left.G]_{n-1}(x) \cdot G\right]_{n+1}(x)-G J_{n}^{2}(x)
$$$$
(-1)^{n}(2)^{n-2} \cdot x^{n-1}(2+x-i) \text {. }
$$

In this paper, we generalized the work of Asci and Gurel in [13] by introducing the Generalized Gaussian Jacobsthal polynomial, Generalized Gaussian Lucas polynomial and thereby we obtain generating function and Cassini identity and introduce $\mathrm{Q}$ matrix for these polynomials. We also present explicit formula and determinantal representation, and give various identities using these polynomials.

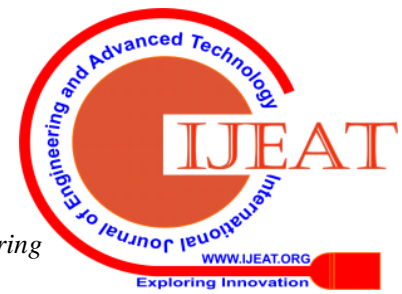




\section{GENERALIZED GAUSSIAN JACOBSTHAL AND GENERALIZED GAUSSIAN JACOBSTHAL LUCAS POLYNOMIALS.}

In this section we give our notions and formulate our main results.

Definition 2.1. The Generalized Gaussian Jacobsthal polynomial $\left\{\mathrm{G}_{\mathrm{k} n \mathrm{n}}(\mathrm{x})\right\}_{\mathrm{n}=0}^{\mathrm{m}=0}$ are defined by the following recurrence relation

$\mathrm{GJ}_{\mathrm{k}_{\mathrm{n}+1}}(\mathrm{x})=\mathrm{GJ}_{\mathrm{k} n \mathrm{n}}(\mathrm{x})+2^{k} \cdot \mathrm{x}_{\mathrm{k} n-1}(\mathrm{x}), \mathrm{n} \geq 1$ and $\mathrm{k}$ is non - negative integer

with initial conditions

$$
\mathrm{GJ}_{\mathrm{k}_{\mathrm{j}} 0}(\mathrm{x})=\frac{\mathrm{i}}{2} \quad \text { and } \quad \mathrm{GJ}_{\mathrm{k}_{\mathbf{\alpha}} 1}(\mathrm{x})=1
$$

Definition 2.2. The Generalized Gaussian Jacobsthal Lucas polynomial $\left\{\mathrm{G}_{\mathrm{k}_{\mathrm{m}} \mathrm{n}}(\mathrm{x})\right\}_{n=0, \pi}^{\mathrm{m}}$ are defined by the following recurrence relation

$$
\mathrm{G}_{\mathrm{k}_{\mathrm{n}+1}+1}(\mathrm{x})=\mathrm{G}_{\mathrm{k}_{\mathrm{n}} \mathrm{n}}(\mathrm{x})+2^{k} \cdot \mathrm{x} \mathrm{G}_{\mathrm{k}_{\mathrm{n}} \mathrm{n}-1}(\mathrm{x}), \mathrm{n} \geq 1 \text {, and } \mathrm{k} \text { is }
$$
non - negative integer

with initial conditions

$$
\mathrm{G}_{\mathrm{k}_{\mathrm{i}, 0}}(\mathrm{x})=2-\frac{\mathrm{i}}{2} \quad \text { and } \quad \mathrm{G}_{\mathrm{k}_{\mathrm{i}} 1}(\mathrm{x})=1+2 \mathrm{x} i \text {. }
$$

It is interesting to note that

$$
\mathrm{G}_{\mathrm{kn} n}(1)=\mathrm{GJ}_{\mathrm{kn}} \quad \text { and } \quad \mathrm{G}_{\mathrm{k}_{\mathrm{n} n} \mathrm{n}}(1)=\mathrm{G}_{\mathrm{k}_{\mathrm{n} n} \mathrm{n}}
$$

where $\mathrm{GJ}_{\mathrm{k} \mathbf{n}}$ and $\mathrm{G}_{\mathrm{k}: \mathrm{n}}$ are the Generalized Gaussian Jacobsthal and Generalized Gaussian Jacobsthal Lucas number.

Next, we give few number of terms of these sequences in the following tables:

\begin{tabular}{|c|c|}
\hline $\mathbf{N}$ & $\mathrm{G}_{\mathrm{j} \mathrm{n}}$ \\
\hline 0 & $2-\frac{i}{2}$ \\
\hline 1 & $1+2 \times x^{x}$ \\
\hline 2 & $\left(1+2^{k+1} \cdot x\right)+i x\left(2-2^{k-1}\right)$ \\
\hline 3 & $\left(1+2^{k+1}+2^{k} \cdot x\right)+i x\left(2-2^{k-1}+2^{k+1} \cdot x\right)$ \\
\hline 4 & $\left(1+2^{k+2} \mathrm{x}+2^{2 k-1} x^{2}\right)+i x\left(2-2^{k-1}+2^{k+2} x-2^{2 k-1} x\right)$ \\
\hline 5 & $\left(1+2^{k+2} \mathrm{x}+2^{2 k+2}{ }_{x} x^{2}+2^{2 k}{ }_{x} x^{2}\right)+i x\left(2-2^{k-1}+2^{k+2} x-2^{2 k}{ }_{x} x+2^{k+1} x-2^{2 k+1} x^{2}\right)$ \\
\hline 6 & $\begin{array}{l}\left(1+2^{k+2} x+2^{2 k+3} \cdot x^{2}+2^{k} \cdot x+2^{3 k+1} \cdot x^{3}\right)+ \\
i x\left(2-2^{k-1}+2^{k+2} x+2^{2 k+1} \cdot x^{2}-2^{2 k-1} x+2^{2 k+3} \cdot x^{2}-2^{3 k-1} \cdot x^{2}\right)\end{array}$ \\
\hline 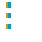 & 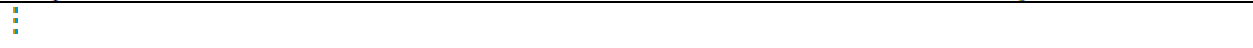 \\
\hline
\end{tabular}

Generalized Gaussian Jacobsthal Polynomials:

\begin{tabular}{|c|c|}
\hline $\mathbf{N}$ & $\mathbf{G J}_{\mathbf{k}_{\mathbf{n}}}$ \\
\hline 0 & $\frac{i}{2}$ \\
\hline 1 & 1 \\
\hline 2 & $1+i 2^{k-1} \cdot \mathrm{X}$ \\
\hline 3 & $\left(1+2^{k} \times\right)+i 2^{k-1} \cdot x$ \\
\hline 4 & $\left(1+2^{k+1} \mathrm{x}\right)+i\left(2^{k-1}+2^{2 k-1}\right) \cdot x$ \\
\hline 5 & $1+\left(2^{k}+2^{k+1}\right) \mathrm{x}+2^{2 k} \cdot x^{2}+i\left(2^{k-1}+2^{2 k-1} \cdot x+2^{2 k-1}\right) \cdot x$ \\
\hline 6 & $1+2^{k+2} \cdot x+\left(2^{2 k}+2^{2 k+1}\right) \cdot x^{2}+i x\left(2^{k-1}+2^{2 k-1} \cdot x+2^{2 k} \cdot x+2^{3 k-1}\right) \times x$ \\
\hline$\vdots$ & $\vdots$ \\
\hline
\end{tabular}

Generalized Gaussian Jacobsthal Lucas Polynomials:

The main recursive properties of the generalized Gaussian Jacobsthal and Generalized Gaussian Lucas polynomial are determined in this section. Initially, we can begin with the characteristic equation, that is

$$
\begin{aligned}
& t^{2}-\mathrm{t}-2^{k} x=0 \\
& \mathrm{t}=\frac{1 \pm \sqrt{1+42^{k} x}}{2} \\
& \mathrm{t}=\frac{1 \pm \sqrt{1+2^{k+2} x}}{2}
\end{aligned}
$$

$\alpha(\mathrm{x})=\frac{1+\sqrt{1+2^{k+2} x}}{2} \quad$ and $\quad \beta(\mathrm{x})=\frac{1-\sqrt{1+2^{k+2} x}}{2}$
$\alpha(\mathrm{x})+\beta(\mathrm{x})=1, \alpha(\mathrm{x}) \cdot \beta(\mathrm{x})=-2^{k}, x, \quad \alpha(\mathrm{x})-\beta(\mathrm{x})=$
$\sqrt{1+2^{k+2} \cdot x}$

\section{Some Properties:}

Theorem 2.1. (i) The Generating function for Generalized Gaussian Jacobsthal polynomial is

$$
\begin{aligned}
& \mathrm{g}(\mathrm{t}, \mathrm{x})=\sum_{\mathrm{n}=0}^{\mathrm{s}} \mathrm{G}_{\mathrm{k}, \mathrm{n}}(\mathrm{x}) \cdot \mathrm{t}^{\mathrm{n}}= \\
& 2 t+i(1-t) \\
& \overline{2-2 t-2^{k+1} t^{2} x}
\end{aligned}
$$

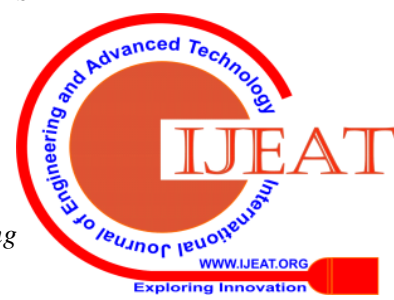


and

(ii) For Generalised Gaussian Jacobthal Lucas polynomial is $\mathrm{h}(\mathrm{t}, \mathrm{x})=\sum_{n=0}^{\mathrm{s}=0} \mathrm{G}_{\mathrm{k}_{\mathrm{k}} \mathrm{n}}(\mathrm{x}) \cdot \mathrm{t}^{\mathrm{n}}=\frac{4-2 \mathrm{t}+\mathrm{i}(\mathrm{t}-1+4 \mathrm{xt})}{2-2 \mathrm{t}-2^{k+1} \mathrm{t}^{2} \mathrm{x}}$

Proof: (i) Let $g(t, x)$ be the generating function of generalized Gaussian Jacobsthal polynomial sequence $\mathrm{GJ}_{\mathrm{k}: \mathrm{n}}(\mathrm{x})$ then

$\mathrm{g}(\mathrm{t}, \mathrm{x})-\mathrm{t} \cdot \mathrm{g}(\mathrm{t}, \mathrm{x})-2^{\mathrm{k}} \cdot \mathrm{x} \cdot \mathrm{t}^{2} \mathrm{~g}(\mathrm{t}, \mathrm{x})=\sum_{\mathrm{n}=0}^{\mathrm{m}} \mathrm{G}_{\mathrm{k} \cdot \mathrm{n}}(\mathrm{x}) \cdot \mathrm{t}^{\mathrm{n}}-$ $\sum_{n=0}^{m} \mathrm{GJ}_{\mathrm{k}_{\mathrm{n}}}(\mathrm{x}) \cdot t^{\mathrm{n}+1}-2^{k} \cdot x \sum_{n=0}^{\mathrm{s}=0} \mathrm{G}_{\mathrm{kn}}(\mathrm{x}) \cdot t^{n+2}$

$$
\begin{aligned}
& =\mathrm{G}_{\mathrm{k}_{0} \mathrm{o}}(\mathrm{x})+\mathrm{G}_{\mathrm{k} \mathbb{1}}(\mathrm{x}) \cdot \mathrm{t}-\mathrm{G}_{\mathrm{k}_{\mathrm{p}} \mathrm{o}}(\mathrm{x}) \mathrm{t} \\
& +\sum_{n=2}^{s e} t^{n}\left[G_{k n}(x)-G_{k n-1}(x)-2^{k} G_{k n-2}(x)\right] \\
& \begin{array}{l}
=\frac{i}{2}+\left(1-\frac{i}{2}\right) t \\
=\frac{2 i+i(1-t)}{2}
\end{array}
\end{aligned}
$$

By taking $\mathrm{g}(\mathrm{t}, \mathrm{x})$ paranthesis, we get

$$
\begin{aligned}
\left(1-t-2^{k} \cdot x \cdot t^{2}\right) g(t, x) & =\frac{2 i+i(1-t)}{2} \\
g(t, x) & =\frac{2 i+i(1-t)}{2\left(1-t-2^{k} x \cdot t^{2}\right)} \\
g(t, x) & =\frac{2 i+i(1-t)}{\left(2-2 t-2^{k+1} x \cdot t^{2}\right)}
\end{aligned}
$$

(ii) Let $\mathrm{h}(\mathrm{t}, \mathrm{x})$ be the generalized function of generalized Gaussian Jacobsthal Lucas polynomial sequence $\mathrm{G}_{\mathrm{k}_{\mathbf{k}} \mathrm{n}}(\mathrm{x})$ then

$$
\begin{aligned}
& \left.\mathrm{h}(\mathrm{t}, \mathrm{x})-\mathrm{t} \cdot \mathrm{h}(\mathrm{t}, \mathrm{x})-2^{\mathrm{k}} \cdot \mathrm{x} \cdot \mathrm{t}^{2} \mathrm{~h}(\mathrm{t}, \mathrm{x})=\sum_{\mathrm{n}=0}^{\mathrm{s}} \mathrm{G}\right]_{\mathrm{k}_{\mathrm{n}} \mathrm{n}}(\mathrm{x}) \cdot \mathrm{t}^{\mathrm{n}}- \\
& \sum_{n=0}^{m} \mathrm{G}_{\mathrm{k} n}(\mathrm{x}) \cdot t^{n+1}-2^{k} \cdot x \sum_{n=0}^{=0} \mathrm{G}_{\mathrm{k} \cdot \mathrm{n}}(\mathrm{x}) \cdot t^{\mathrm{n}+2} \\
& =\mathrm{G}_{\mathrm{k}_{j} 0}(\mathrm{x})+\mathrm{G}_{\mathrm{k}_{\mathrm{k} 1} 1}(\mathrm{x}) \cdot \mathrm{t}-\mathrm{G}_{\mathrm{k}_{\mathrm{j}, 0}}(\mathrm{x}) \mathrm{t} \\
& \sum_{n=2}^{m} t^{n}\left[G_{k n}(x)-G_{k n-1}(x)-2^{k} G_{k n-2}^{m}(x)\right] \\
& =2-\frac{i}{2}+(1+2 x i)-\left(2-\frac{i}{2}\right) t \\
& =2-\frac{i}{2}+1+2 x i-2 \mathrm{t}+\frac{i}{2} t \text {, }
\end{aligned}
$$

that is,

$\left(1-t-2^{k} \cdot x \cdot t^{2}\right) h(t, x)=\frac{4-i-2 t-4 x t i-4 t+i t}{2}$

which implies,

$$
\begin{array}{ll}
h(t, x) & =\frac{4-2 t+i(4 x t-1+t)}{2\left(1-t-2^{2} x_{x x} t^{2}\right)} \\
\text { and hence } & h(t, x)=\frac{4-2 t+i(4 x t-1+t)}{2-2 t-2^{k+1} x \cdot t^{2}}
\end{array}
$$

The following results hold immediately by applying induction:

\section{Theorem 2.2. (Generalized Binet Formula)}

For $\mathrm{n} \geq 0$

$$
\begin{aligned}
& \text { (i) } \mathrm{GJ}_{\mathrm{k}_{\mathrm{i}} \mathrm{n}}(\mathrm{x})=\frac{\mathrm{a}^{\mathrm{n}}(\mathrm{x})-\beta^{\mathrm{n}}(\mathrm{x})}{\alpha(x)-\beta(x)}+\mathrm{i} \cdot \mathrm{x} \cdot 2^{\mathrm{k}-1} \frac{a^{\mathrm{n}-1}(x)-\beta^{\mathrm{n}-1}(x)}{a(x)-\beta(x)} \\
& \text { (ii) (i) } G_{k_{k n}}(x)=\alpha^{n}(x)+\beta^{n}(x) \quad+ \\
& \text { 1. } x \cdot 2^{\mathrm{k}-1}\left(\alpha^{\mathrm{n}-1}(x)+\beta^{\mathrm{n}-1}(x)\right)
\end{aligned}
$$

Theorem 2.3. The explicit formula of Generalized Gaussian Jacobsthal polynomial is

$$
\begin{aligned}
& \mathrm{GJ}_{\mathrm{kn}}(\mathrm{x})=\sum_{y=0}^{\left[\frac{n-1}{2}\right]} \cdot\left(\begin{array}{c}
n-\gamma-1 \\
r
\end{array}\right)\left(2^{k} \cdot x\right)^{\gamma}+\mathrm{i} \sum_{\gamma=0}^{\left[\frac{n-2}{2}\right]} \cdot\left(\begin{array}{c}
n-\gamma-2 \\
r
\end{array}\right) \\
& \left(2^{k}\right)^{\gamma} \cdot(x)^{\gamma+1}
\end{aligned}
$$

Theorem 2.4. The explicit formula of Generalized Gaussian Jacobsthal Lucas polynomial is

$\mathrm{G}_{\mathrm{k}_{\mathrm{n}} \mathrm{n}}(\mathrm{x})=\sum_{y=0}^{\left\lfloor\frac{n}{2}\right]} \frac{n}{n-r}\left(\begin{array}{c}n-r \\ y\end{array}\right)\left(2^{k} \times x\right)^{r}+\mathrm{i} \sum_{y=0}^{\left[\frac{n-1}{2}\right]} \frac{n-1}{n-r-1}\left(\begin{array}{c}n-y-1 \\ y\end{array}\right)$ $\left(2^{k}\right)^{r} \cdot(x)^{r+1}$

Theorem 2.5. Let $D_{n}(x)$ denote the $\mathrm{n} \times n$ tridiagonal matrix as

$$
D_{n}(x)=\left[\begin{array}{ccccc}
1 & i 2^{k-1} & 0 & \cdots & 0 \\
-x & 1 & 2^{k} x & \vdots & \vdots \\
0 & -1 & 1 & \vdots & 0 \\
\vdots & \vdots & \vdots & : & 2^{k} x \\
0 & 0 & 0 & -1 & 1
\end{array}\right], \mathrm{n} \geq 1
$$

and let $D_{0}(x)=\frac{i}{2}$, Then $\operatorname{det} D_{n}(x)=\mathrm{G}_{\mathrm{k}_{n} \mathrm{n}}(\mathrm{x}), \mathrm{n} \geq 1$

Proof: We shall prove the result by using mathematical induction on $\mathrm{n}$.

For $\mathrm{n}=1$ and $\mathrm{n}=2$

$$
\operatorname{det} D_{1}(x)=1=\mathrm{G}_{\mathrm{k}_{1} 1}(\mathrm{x})
$$$$
\text { and } \quad \operatorname{det} D_{2}(x)=1+i 2^{k-1} x=\mathrm{GJ}_{\mathrm{k}_{2}}(\mathrm{x})
$$

Assume that the theorem is true for $n-1$ and $n-2$, that is

$$
\begin{aligned}
& \operatorname{det} D_{n-1}(x)=\mathrm{G}_{\mathrm{k} n-1}(\mathrm{x}) \\
& \text { and } \quad \operatorname{det} D_{n-2}(x)=\mathrm{G}_{\mathrm{k}_{\mathrm{n}-2}}(\mathrm{x})
\end{aligned}
$$

Now, we shall prove that the theorem for $n$

For,

$$
\text { det } \begin{aligned}
D_{n}(x) & =\operatorname{det} D_{n-1}(x)+2^{k} \cdot x \operatorname{det} D_{n-2}(x) \\
& =G_{k_{n}-1}(x)+2^{k} \cdot x \mathrm{G}_{\mathrm{k} n-2}(\mathrm{x}) \\
& =G_{k_{n} n}(x)
\end{aligned}
$$

Theorem 2.6. Let $H_{n}(x)$ denote the $\mathrm{n} \times n$ tridiagonal matrix as

$$
H_{n}(x)=\left[\begin{array}{ccccc}
2-\frac{i}{2} & \frac{x}{2}-1 & 0 & \cdots & 0 \\
1 & i x & 2^{k} x & \vdots & \vdots \\
0 & -1 & 1 & \vdots & 0 \\
\vdots & \vdots & \vdots & \ddots & 2^{k} x \\
0 & 0 & 0 & -1 & 1
\end{array}\right], \mathrm{n} \geq 1
$$

Then, det $H_{\mathrm{n}}(x)=\mathrm{G}_{\mathrm{kn}-1}(\mathrm{x}), \mathrm{n} \geq 0$.

Proof: We shall prove the result by using mathematical induction on $\mathrm{n}$.

For $\mathrm{n}=1$ and $\mathrm{n}=2$

$$
\begin{array}{ll}
\operatorname{det} H_{1}(x) & =2-\frac{i}{2}=\mathrm{G}_{\mathrm{k}_{i} 0}(\mathrm{x}) \\
\text { and } \quad & \operatorname{det} H_{2}(x)=1+2 \mathrm{x} i=\mathrm{G}_{\mathrm{k}_{2} 1}(\mathrm{x})
\end{array}
$$

Assume that the theorem is true for $n-1$ and $n-2$

$$
\begin{aligned}
\operatorname{det} H_{n-1}(x) & =\mathrm{G}_{\mathrm{k}_{\mathrm{k}-2}}(\mathrm{x}) \\
\text { and } \quad \operatorname{det} H_{n-2}(x) & =\mathrm{G}_{\mathrm{k}_{\mathrm{k} n-1}}(\mathrm{x})
\end{aligned}
$$

Now, we shall prove that the theorem for $\mathrm{n}$ For,

$$
\text { det } \begin{aligned}
H_{n}(x) & =\operatorname{det} H_{n-1}(x)+2^{k}{ }_{x} x \operatorname{det} H_{n-2}(x) \\
& =\mathrm{G}_{\mathrm{k}_{\mathrm{n}-1}}(\mathrm{x})+2^{k}{ }_{x} x \mathrm{G}_{\mathrm{k}_{\mathrm{n}-2}}(\mathrm{x}) \\
& =G_{k_{k_{n}}}(x)
\end{aligned}
$$

Now, we introduce the matrices $\mathrm{Q}(\mathrm{x}), \mathrm{P}$ and $\mathrm{R}$ that plays the role of the Q-Matrix of Fibonacci numbers. Let $\mathrm{Q}(\mathrm{x}), \mathrm{P}$ and $\mathrm{R}$ denotes the $2 \times 2$ matrices defined as

$$
\begin{aligned}
& \mathrm{Q}(\mathrm{x})=\left[\begin{array}{cc}
1 & 2^{k} x \\
1 & 0
\end{array}\right], \quad \mathrm{P}=\left[\begin{array}{cc}
1+i x 2^{k-1} & 1 \\
1 & \frac{1}{2}
\end{array}\right], \mathrm{R}= \\
& {\left[\begin{array}{cc}
1+2^{k+1} x+i x\left(2-2^{k-1}\right) & 1+2 i x \\
1+2 i x & 2-\frac{i}{2}
\end{array}\right] .}
\end{aligned}
$$

Theorem 2.7. Let $\mathrm{n} \geq 1$, Then

$$
Q^{n}(x) \cdot P=\left[\begin{array}{cc}
G]_{k_{n} n+2}(x) & G_{k_{n}+1}(x) \\
G_{k_{n} n+1}(x) & G_{k_{n} n}(x)
\end{array}\right]
$$

where $\mathrm{GJ}_{\mathrm{k}_{\mathrm{n}}}(\mathrm{x})$ is the $\mathrm{nth}$ Generalized Gaussian Jacobsthal Polynomial.

Proof:- We shall prove the result by using mathematical induction on $\mathrm{n}$.

For $\mathrm{n}=1$

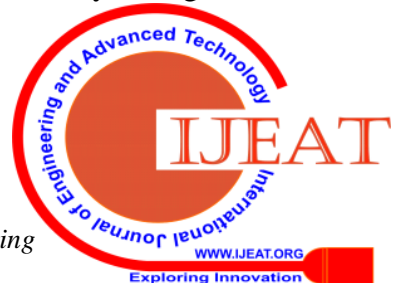




$$
\begin{aligned}
\mathrm{Q}(\mathrm{x}) \cdot \mathrm{P} & =\left[\begin{array}{cc}
1 & 2^{k} x \\
1 & 0
\end{array}\right]\left[\begin{array}{cc}
1+i x 2^{k-1} & 1 \\
1 & \frac{i}{2}
\end{array}\right] \\
& =\left[\begin{array}{cc}
1+i 2^{k-1} \cdot x+2^{k} x & 1+i 2^{k-1} x \\
1+i 2^{k-1} x & 1
\end{array}\right] \\
& =\left[\begin{array}{ll}
\mathrm{GJ}_{\mathrm{k}_{2} 3}(\mathrm{x}) & \mathrm{GJ}_{\mathrm{k}_{2} 2}(\mathrm{x}) \\
\mathrm{G}_{\mathrm{k}_{2} 2}(\mathrm{x}) & \mathrm{G}_{\mathrm{k}_{2} 1}(\mathrm{x})
\end{array}\right]
\end{aligned}
$$

Assume that the theorem hold for $\mathrm{n}=\mathrm{t}$, that is

$$
\begin{aligned}
{[Q(x)]^{t} \cdot P } & =\left[\begin{array}{cc}
1 & 2^{k} x \\
1 & 0
\end{array}\right]^{t}\left[\begin{array}{cc}
1+i x 2^{k-1} & 1 \\
1 & \frac{i}{2}
\end{array}\right] \\
& =\left[\begin{array}{cc}
G_{k_{i} t+2}(x) & G_{k_{t} t+1}(x) \\
G_{k_{t} t+1}(x) & \mathrm{G}_{\mathrm{k}_{t} t}(\mathrm{x})
\end{array}\right]
\end{aligned}
$$

Now, we shall prove the theorem for $\mathrm{n}=\mathrm{t}+1$, we have

$$
\begin{aligned}
& {[\mathrm{Q}(\mathrm{x})]^{\mathrm{t+1}} \cdot \mathrm{P}=\left[\begin{array}{cc}
1 & 2^{k} x \\
1 & 0
\end{array}\right]^{t+1}\left[\begin{array}{cc}
1+i \mathrm{x} 2^{k-1} & 1 \\
1 & \frac{i}{2}
\end{array}\right]} \\
& =\left[\begin{array}{cc}
1 & 2^{k} x \\
1 & 0
\end{array}\right]\left[\begin{array}{cc}
1 & 2^{k} x \\
1 & 0
\end{array}\right]^{t}\left[\begin{array}{cc}
1+i x 2^{k-1} & 1 \\
1 & \frac{1}{2}
\end{array}\right] \\
& =\left[\begin{array}{cc}
1 & 2^{k} x \\
1 & 0
\end{array}\right] \cdot\left[\begin{array}{cc}
\mathrm{G}_{\mathrm{k}_{\mathrm{t}} \mathrm{t}+2}(\mathrm{x}) & \mathrm{G}_{\mathrm{k}_{\mathrm{t}} t+1}(\mathrm{x}) \\
\mathrm{GJ}_{\mathrm{k}_{\mathrm{t} t+1}(\mathrm{x})} & \mathrm{G}_{\mathrm{k}_{\mathrm{t}} \mathrm{t}}(\mathrm{x})
\end{array}\right] \\
& =\left[\begin{array}{ll}
\mathrm{G}_{\mathrm{k}_{i} t+\mathrm{a}}(\mathrm{x}) & \mathrm{GJ}_{\mathrm{k}_{i} t+2}(\mathrm{x}) \\
\mathrm{GJ}_{\mathrm{k}_{i} t+2}(\mathrm{x}) & \mathrm{GJ}_{\mathrm{k}_{i} t+1}(\mathrm{x})
\end{array}\right]
\end{aligned}
$$

Theorem 2.8. Let $\mathrm{n} \geq 1$, Then

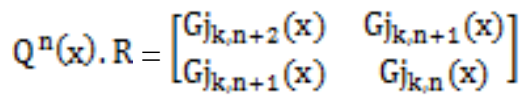

where $\mathrm{G}_{\mathrm{k}_{\mathrm{n}} \mathrm{n}}(\mathrm{x})$ is the $\mathrm{nth}$ Generalized Gaussian Jacobsthal Lucas Polynomial.

Proof: We shall prove the result by using mathematical induction on $\mathrm{n}$.

For $\mathrm{n}=1$

$$
\mathrm{Q}(\mathrm{x}) \cdot \mathrm{R}=\left[\begin{array}{cc}
1 & 2^{k} x \\
1 & 0
\end{array}\right] \cdot\left[\begin{array}{cc}
1+2^{k+1} \cdot x+i x\left(2-2^{k-1}\right) & 1+2 i x \\
1+2 i x & 2-\frac{1}{2}
\end{array}\right]
$$$$
\left[\begin{array}{cc}
1+2^{k+1} \cdot x+2^{k} \cdot x+i x\left(2-2^{k-1}+2^{k+1} x\right) & 1+2 i x+2^{k+1} x-i \cdot 2^{k-1} \cdot x \\
1+2 i x+2^{k+1} x-i \cdot 2^{k-1} \cdot x & 1+2 x i
\end{array}\right]
$$

\section{Theorem 2.10. For $n \geq 1$}

$$
\left[\begin{array}{ll}
G_{k_{k} t+a}(x) & G_{k_{i} t+2}(x) \\
G_{k_{k} t+2}(x) & G_{k_{j} t+1}(x)
\end{array}\right]
$$

\section{Theorem 2.9. (Generalized Cassini Identity)}

For $\mathrm{n} \geq 1$

$$
(-1)^{n}\left(2^{k}\right)^{n-2} \cdot x^{n-1}\left(2^{k-1} x+2-1\right)
$$

Proof: We can prove the theorem by matrix method. For, first of all we shall determine the determinants of matrices.

$-\frac{1}{2}\left[2^{k-1} \cdot x+2-i\right]$

$$
\begin{aligned}
& \operatorname{det} Q^{n-1}(x)=\left|\begin{array}{cc}
1 & 2^{k} \cdot x \\
1 & 0
\end{array}\right|^{n-1}=\left(-2^{k} x\right)^{n-1} . \\
& \operatorname{det} P=\left|\begin{array}{cc}
1+i \cdot 2^{k-1} \cdot x & \frac{1}{i} \\
1 & \frac{i}{2}
\end{array}\right|=\frac{i}{2}-\frac{2^{k-1}}{2} x-2=
\end{aligned}
$$

By Theorem 2.7,

$$
[\mathrm{Q}(\mathrm{x})]^{\mathrm{n}-1} \cdot \mathrm{p}=\left[\begin{array}{cc}
\mathrm{G}_{\mathrm{k} n+1}(\mathrm{x}) & \mathrm{G}_{\mathrm{k}_{n} \mathrm{n}}(\mathrm{x}) \\
\mathrm{G}_{\mathrm{k}_{n} \mathrm{n}}(\mathrm{x}) & \mathrm{G}_{\mathrm{k}_{n} \mathrm{n}-1}(\mathrm{x})
\end{array}\right]
$$

We get the determinants of the matrices

$$
\begin{array}{r}
\left.G J_{k, n-1}(x) \cdot G J_{k, n+1}(x)-G\right]_{k, n}^{2}(x)=\operatorname{det}\left(Q^{n-1}(x) \cdot P\right) \\
=\operatorname{det}\left(Q^{n-1}(x)\right) \cdot \operatorname{det}(P) \\
\left.=(-2)^{k} \cdot x\right)^{n-1} \cdot\left(-\frac{1}{2}\left[2^{k-1} \cdot x+2-i\right]\right) \\
=(-1)^{n} \cdot\left(2^{k}\right)^{n-2} \cdot x^{n-1}\left(2^{k-1} \cdot x+2-i\right)
\end{array}
$$

$G_{k_{2} n-1}(x) \cdot G_{k_{n} n+1}(x)-G_{k_{n} n}^{2}(x)$

$(-1)^{n-1} \cdot 2^{n-2} \cdot x^{n-1}\left[(8 x)^{2}+\left(2+3.2^{k-1}\right) x+2-\right.$

$\left.i 2^{k+2} \cdot x\right]$

Proof: We can prove the theorem by matrix method. For, first $\left[\begin{array}{cc}1+2^{k+1} \cdot x+2^{k} \cdot x+i x\left(2-2^{k-1}+2^{\mathrm{k}+1} x\right) & 1+2^{\mathrm{k}+1} x+i \cdot x\left(2-2^{k}\right. \\ 1+2^{\mathrm{k}+1} x+i \cdot x\left(2-2^{k-1}\right) & 1+2 x i\end{array}\right.$

of_allywe determine the determinants of matrices.

$$
=\left[\begin{array}{ll}
\mathrm{G}_{\mathrm{k}_{\mathrm{w}} \mathrm{a}}(\mathrm{x}) & \mathrm{G}_{\mathrm{k}_{2} 2}(\mathrm{x}) \\
\mathrm{G}_{\mathrm{k}_{\mathrm{z}} 2}(\mathrm{x}) & \mathrm{G}_{\mathrm{k}_{1} 1}(\mathrm{x})
\end{array}\right]
$$

Assume that the theorem holds for $\mathrm{n}=\mathrm{t}$, that is $[\mathrm{Q}(\mathrm{x})]^{\mathrm{t}}$

$$
\begin{aligned}
& {\left[\begin{array}{cc}
1 & 2^{k} x \\
1 & 0
\end{array}\right]^{t} } {\left[\begin{array}{cc}
1+2^{k+1} x+i x\left(2-2^{k-1}\right) & 1+2 i x \\
1+2 i x & 2-\frac{i}{2}
\end{array}\right] } \\
&=\left[\begin{array}{cc}
G_{k_{i} t+2}(x) & G_{k_{i} t+1}(x) \\
G_{k_{i} t+1}(x) & G_{k_{i} t}(x)
\end{array}\right]
\end{aligned}
$$

$. \mathrm{R}=$

$$
\begin{gathered}
\operatorname{det} Q^{n-1}(x)=\left|\begin{array}{cc}
1 & 2^{k} \cdot x \\
1 & 0
\end{array}\right|^{n-1}=\left(-2^{k} x\right)^{n-1} \\
\operatorname{detR}=\left|\begin{array}{cc}
1+2^{k+1} \cdot x+i x\left(2-2^{k-1}\right) & 1+2 i x \\
1+2 i x & 2-\frac{i}{2}
\end{array}\right| \\
=\left[(B x)^{2}+\left(2+3.2^{k-1}\right) x+2-i-i 2^{k+2^{2}} \cdot x\right]
\end{gathered}
$$

By Theorem 2.8,

$$
\mathrm{Q}^{\mathrm{n}-1}(\mathrm{x}) \cdot \mathrm{R}=\left[\begin{array}{cc}
\mathrm{G}_{\mathrm{k}_{\mathrm{n} n+1}}^{\mathrm{J}}(\mathrm{x}) & \mathrm{G}_{\mathrm{k}_{\mathrm{n}} \mathrm{n}}(\mathrm{x}) \\
\mathrm{G}_{\mathrm{k}_{\mathrm{n}} \mathrm{n}}(\mathrm{x}) & \mathrm{G}_{\mathrm{k}_{\mathrm{n}-1}}(\mathrm{x})
\end{array}\right]
$$

We get the determinants of the matrices

$$
\begin{aligned}
G_{k_{2} n-1}(x) \cdot G_{j_{k_{2} n+1}}(x)-G_{k_{l} n}^{2}(x) & =\operatorname{det}\left(Q^{n-1}(x) \cdot \mathrm{R}\right) \\
& =\operatorname{det}\left(Q^{n-1}(x)\right) \cdot \operatorname{det}(\mathrm{R})
\end{aligned}
$$

Now, we shall prove that the theorem for $n=t+1$, For, 


$$
\begin{aligned}
& \quad= \\
& \begin{array}{l}
\left.(-2)^{k} \cdot x\right)^{n-1} \cdot \frac{1}{2}\left[(8 x)^{2}+\left(2+3.2^{k-1}\right) x+2-i-\right. \\
\left.i 2^{k+2} \cdot x\right]
\end{array} \\
& \quad= \\
& \begin{array}{l}
(-1)^{n-1} \cdot 2^{n-2} \cdot x^{n-1}\left[(8 x)^{2}+\left(2+3.2^{k-1}\right) x+2-i-\right. \\
\left.i 2^{k+2} \cdot x\right]
\end{array}
\end{aligned}
$$

Theorem 2.11. The sums of the Generalized Gaussian Jacobsthal Polynomial and Generalized Gaussian Jacobsthal Lucas Polynomial are given as:
(i) $\sum_{m=0}^{n} G J_{k m}(x)=\frac{1}{2^{k} x}\left[G j_{k n+2}(x)-1\right]$;
(ii) $\sum_{m=0}^{n} G_{j_{k m} m}(x)=\frac{1}{2^{k_{x}}}\left[G_{j_{k n+2}}(x)-(1+2 x i)\right]$.

Proof: (i) For $n \geq 1$, we have

$$
\begin{aligned}
& \mathrm{GJ}_{\mathrm{k} n+1}(\mathrm{x})=\mathrm{G}_{\mathrm{k} n}(\mathrm{x})+2^{k} \cdot \mathrm{x}_{\mathrm{k}_{n} \mathrm{n}-1}(\mathrm{x}), \\
& \mathrm{GJ}_{\mathrm{k}_{\mathrm{n}-1}}(\mathrm{x})=\frac{1}{2^{k_{x}}}\left[\mathrm{GJ}_{\mathrm{kn}+1}(\mathrm{x})-\mathrm{GJ}_{\mathrm{k}_{\mathrm{n}} \mathrm{n}}(\mathrm{x})\right] \text {. }
\end{aligned}
$$

For the last equation

$$
\begin{aligned}
& \mathrm{GJ}_{\mathrm{k}_{\mathrm{p}} 0}(\mathrm{x})=\frac{1}{2^{k_{x}}}\left[\mathrm{GJ}_{\mathrm{k}_{2}}(\mathrm{x})-\mathrm{GJ}_{\mathrm{k}_{1}}(\mathrm{x})\right] \text {, } \\
& \mathrm{G}_{\mathrm{k}_{2} 1}(\mathrm{x})=\frac{1}{2^{k_{x}}}\left[\mathrm{GJ}_{\mathrm{k}_{\mathrm{a}}}(\mathrm{x})-\mathrm{GJ}_{\mathrm{k}_{2}}(\mathrm{x})\right] \text {, } \\
& \mathrm{G}_{\mathrm{k}_{2} 2}(\mathrm{x})=\frac{1}{2^{\mathrm{k}_{x}}}\left[\mathrm{GJ}_{\mathrm{k} 4}(\mathrm{x})-\mathrm{GJ}_{\mathrm{ka}_{\mathrm{a}}}(\mathrm{x})\right] \text {, } \\
& \begin{array}{l}
\mathrm{GJ}_{\mathrm{kn}-1}(\mathrm{x})=\frac{1}{2^{k} x_{x}}\left[\mathrm{G}_{\mathrm{kn+1}}(\mathrm{x})-\mathrm{GJ}_{\mathrm{knn}}(\mathrm{x})\right], \\
\mathrm{GJ}_{\mathrm{kn}}(\mathrm{x})=\frac{1}{2}\left[\mathrm{G}_{\mathrm{kn+2}}(\mathrm{x})-\mathrm{G}_{\mathrm{kn+1}}(\mathrm{x})\right] .
\end{array}
\end{aligned}
$$

On adding all of the above equation, can get

$$
\begin{gathered}
\left.\left.\left.\sum_{m=0}^{n} G\right]_{k m}(x)=\frac{1}{2^{k_{x} x}}[G]_{k n+2}(x)-G\right]_{k_{i} 1}(x)\right] \\
\left.=\frac{1}{2^{k_{x} x}}[G]_{k_{n+2}}(x)-1\right] .
\end{gathered}
$$

(ii) For $\mathrm{n} \geq 1$, we have

$$
\begin{aligned}
& \mathrm{G}_{\mathrm{k} n+1}(\mathrm{x})=\mathrm{G}_{\mathrm{k}_{\mathrm{k} n}}(\mathrm{x})+2^{k} \cdot \mathrm{x} \mathrm{G}_{\mathrm{k} n-1}(\mathrm{x}) \text {; } \\
& \mathrm{G}_{\mathrm{k}_{\mathrm{n}-1}}(\mathrm{x})=\frac{1}{2^{\mathrm{k}_{\mathrm{x}}}}\left[\mathrm{G}_{\mathrm{k}_{\mathrm{n}+1}}(\mathrm{x})-\mathrm{G}_{\mathrm{k}_{\mathrm{n}} \mathrm{n}}(\mathrm{x})\right] \text {. }
\end{aligned}
$$

For the last equation

$$
\begin{aligned}
& \mathrm{G}_{\mathrm{k}_{2} 0}(\mathrm{x})=\frac{1}{2^{k} x}\left[\mathrm{G}_{\mathrm{k}_{2} 2}(\mathrm{x})-\mathrm{G}_{\mathrm{k}_{\mathrm{\alpha}} 1}(\mathrm{x})\right] \text {, } \\
& \mathrm{G}_{\mathrm{k}_{2} 1}(\mathrm{x})=\frac{1}{2^{k_{x} x}}\left[\mathrm{G}_{\mathrm{k}_{\mathrm{z}} \mathrm{a}}(\mathrm{x})-\mathrm{G}_{\mathrm{k}_{\mathrm{\alpha}} 2}(\mathrm{x})\right] \text {, } \\
& \mathrm{G}_{\mathrm{k}_{2} 2}(\mathrm{x})=\frac{{ }_{1}}{2^{\mathrm{k}_{x} x}}\left[\mathrm{G}_{\mathrm{k}_{2} 4}(\mathrm{x})-\mathrm{G}_{\mathrm{k}_{\mathrm{x}} \mathrm{I}}(\mathrm{x})\right] \text {, } \\
& \begin{array}{l}
\mathrm{G}_{\mathrm{kn} n-1}(\mathrm{x})=\frac{1}{2^{k_{x}}}\left[\mathrm{G}_{\mathrm{k}_{\mathrm{n}+1}}(\mathrm{x})-\mathrm{G}_{\mathrm{kn}}(\mathrm{x})\right], \\
\mathrm{G}_{\mathrm{k}_{\mathrm{n} n}}(\mathrm{x})=\frac{1}{2^{k_{x}}}\left[\mathrm{G}_{\mathrm{k}_{\mathrm{n}+2}}(\mathrm{x})-\mathrm{G}_{\mathrm{kn+1}}(\mathrm{x})\right] .
\end{array}
\end{aligned}
$$

On adding all of the above equation, can get

$$
\begin{aligned}
& \sum_{m=0}^{n} G_{k_{k} m}(x)=\frac{1}{2^{k_{x}} x}\left[G_{j_{k_{n} n+2}}(x)-G_{j_{k_{\alpha}}}(x)\right] \text {, } \\
& =\frac{1}{2^{k_{x}}}\left[G_{f_{k n+2}}(x)-(1+2 x i)\right] \text {. }
\end{aligned}
$$

\section{REFERENCES}

[1] Asci M., Gurel E. "Bivariate Gaussian Fibonacci and Lucas polynomial" (to appear in Ars. Comb).

[2] Asci M., Gurel E. "Gaussian Jacobsthal and Gaussian Jacobsthal Lucas Numbers" (to appear in Ars. Comb).

[3] Asci M., Gurel E. "Gaussian Jacobsthal and Gaussian Jacobsthal Lucas Polynomials, Notes on Number Theory and Discrete Mathematics. Vol. 19, 2013, No. 1, 25-36.

[4] Berzsenyi G., Gaussian Fibonacci numbers, Fibonacci Quarterly, Vol. 15, 1977, No. 3, 233-236.

[5] Good I. J., Complex Fibonacci and Lucas numbers, continued fractions, and the square root of the golden ratio. Fibonacci Quarterly, Vol. 31, 1993, No. 1, 7-20.
[6] Harman C. J., Complex Fibonacci Numbers. Fibonacci Quarterly, Vol. 19, 1981, No. 1, 82-86.

[7] Horadam A.F., Generating Functions for Powers of a Certain Generalized Sequence of Number. Duke Math. J., Vol. 32, 1965, 437-46.

[8] Horadam A.F., A Complex Fibonacci Numbers and Fibonacci Quaternions. American Math. Monthly, Vol. 70, 1963, 289-291.

[9] Horadam A.F., Jacobsthal Representation Numbers, Fibonacci Quarterly, Vol. 34, 1996, 40-54.

[10] Horadam A.F., A Gemeralized Fibonacci Sequence, American Math. Monthly, Vol. 68, 1961, 455-459.

[11] Horadam A.F., E.M. Horadam. Roots of recurrence-generated polynomials. Fibonacci Quarterly, Vol. 20, 1982, No. 3, 219-226.

[12] Jordan J.H., Gaussian Fibonacci and Lucas numbers. Fibonacci Quarterly, Vol. 3, 1965, 315-318.

[13] Pethe S., Horadam. A.F., Generalized Gaussian Fibonacci numbers. Bull Austral. Math. Soc., Vol. 33, 1986, No. 1, 37-48. 